\title{
An analytical approach to deformation of anisotropic ice-crystal aggregates
}

\author{
Throstur Thorsteinsson \\ Department of Earth and Space Sciences, University of Washington, Seattle, Washington 98195-1310, U.S.A.
}

\begin{abstract}
Deformation rates of single hexagonal crystals, deforming by glide on the basal plane, are described as a function of stress state and crystal orientation. These results are used to infer the deformation rate of crystal aggregates assuming that the stress distribution within the crystal aggregate is homogeneous. Analytical equations for the deformation rate of anisotropic ice aggregates are derived for vertically symmetric girdle fabric. This type of fabric is approximated by a uniform distribution of $c$-axis orientations between a cone angle and a smaller girdle angle relative to the symmetry axis. For simple shear stress acting on a single-maximum fabric there is a slight de-enhancement for cone angles of $60-90^{\circ}$. In uniaxial compression a maximum enhancement of $\sim 1.7$ occurs at a cone angle of $57^{\circ}$. A pure shear stress state has similar features, with the additional complication that it causes a non-zero transverse strain rate, except for perfect vertical alignment of crystals and isotropic fabric. In combined states of stress the contribution of each stress component to the strain rate depends on fabric. A single enhancement factor is not adequate to describe the effects of anisotropy for complex stress states.
\end{abstract}

\section{INTRODUGTION}

For more than 40 years, ice deformation has generally been modeled using Glen's flow law (Glen, 1958). Due to its success in explaining early field observations, combined with its simple analytical formulation, only minor adjustments have been made to Glen's law since its introduction. Recently, as measurement techniques and data quality have improved, important discrepancies have emerged. The deformation rate of boreholes (Gundestrup and Hansen, 1984; DahlJensen and Gundestrup, 1987; Thorsteinsson and others, 1999), for instance, is often very different from what Glen's flow law predicts (Paterson, 1991). This has led to a commonly used correction to Glen's law, expressed by empirical scalar "enhancement factors".

In an ice sheet, an initially isotropic polycrystalline ice aggregate undergoing ductile deformation will develop lattice-preferred orientation (fabric) as a result of intracrystalline slip (Wenk and Christie, 1991). At near-melting temperatures, recrystallization also contributes to the fabric evolution (Duval and Castelnau, 1995). Fabric has been well documented in ice sheets by extensive thin-section measurements on ice from ice cores (Alley and others, 1995; Gow and others, 1997; Thorsteinsson and others, 1997), and sonic logging both in boreholes and on the ice cores themselves (Kohnen and Gow, 1979; Taylor, 1982; Anandakrishnan and others, 1994; Thorsteinsson and others, 1999). A consequence of fabric development is that bulk physical properties become anisotropic, as shown by experiments and theory (Steinemann, 1958; Russell-Head and Budd, 1979; Duval, 1981; Duval and le Gac, 1982; Budd and Jacka, 1989; Van der Veen and Whillans, 1990; Alley, 1992; Anandakrishnan and others, 1994; Azuma, 1994, 1995; Azuma and Goto-Azuma, 1996; Castelnau and others, 1996). At Dye 3 in Greenland, fabric accounts for roughly $70 \%$ of the difference between measured deformation rates and those predicted by Glen's flow law (Thorsteinsson and others, 1999; Cuffey and others, 2000). (Glen, 1958) noted that strong fabric would compromise all theories based on isotropy.

The goal of this paper is to investigate the non-linear deformation of anisotropic polycrystalline ice in various combinations of stress states and with prescribed fabric similar to those found in ice sheets. I use the homogeneousstress assumption (often referred to as the Sachs model (Sachs, 1928)) to formulate an analytical anisotropic flow law. This method displays the connection between singlecrystal deformation and bulk behavior clearly, and allows convenient exploration of the bulk response for representative fabrics in various stress states. The non-linearity of the flow law is included in the analysis and leads to a non-linear coupling of deviatoric stress components in the bulk.

I begin by examining the deformation rate of single crystals deforming by glide on the basal plane. The characteristics of the single-crystal deformation rate are displayed in a new way to yield insight into the bulk deformation. A stress exponent of $n=3$ is assumed, but any value of $n$ is easily accommodated. The development allows for any fabric specified by an orientation distribution function. I assume that the specified fabric has a vertical axis of rotational symmetry, as is commonly found over large depth ranges in ice cores (Herron and others, 1985; Gow and others, 1997; Thorsteinsson and others, 1997), and that it can be described by a girdle fabric. Girdle fabric (Fig. 1) is defined by two parameters: a cone angle $\alpha$ which is a half-apex angle of a cone within which all the crystals are uniformly distributed, and a girdle angle $\alpha_{0}$, which is the angle of a smaller cone within which no crystals are found. I explore the bulk deformation in several commonly encountered stress states, and derive relatively simple analytical equations for the deformation rate. The cone and girdle angles are the only variables needed in addition to the 


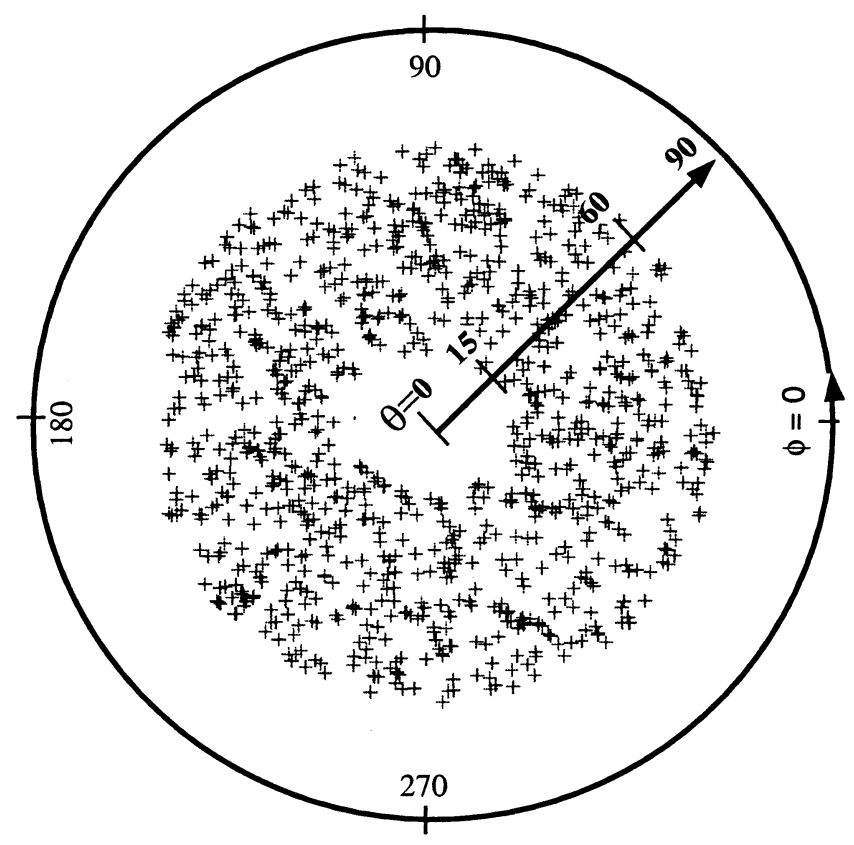

Fig. 1. An equal-area plot (Schmidt plot) of a girdle fabric. The $c$ axes are distributed uniformly between $\alpha_{0}=15^{\circ}$ (girdle angle) and $\alpha=60^{\circ}$ (cone angle). Also shown are the zenith angle, $\theta$, and the azimuth, $\phi$.

variables required to model isotropic ice using Glen's flow law. Examples of how the anisotropy affects ice flow are used to point out differences between anisotropic and isotropic materials, and highlight the importance of accounting for the anisotropy. These equations can be used to separate the effects of anisotropy from other factors responsible for the deformation of boreholes (Thorsteinsson and others, 1999; Cuffey and others, 2000). In addition, these equations can be used to explore how much "softer" or "stiffer" the fabric makes the ice in a given stress field. In this paper, I focus on the properties of ice $\left(\mathrm{I}_{\mathrm{h}}\right)$ crystals, but the formulation is general enough to apply to other anisotropic materials.

\section{BAGKGROUND}

Hexagonal ice crystals (ice $I_{h}$ ) show very strong plastic anisotropy. Ice $\mathrm{I}_{\mathrm{h}}$ deforms almost entirely by dislocation glide on the basal plane, other slip systems being 100 times harder (Duval and others, 1983). To relate the deformation of single crystals to bulk deformation, assumptions about the distribution of stress and strain within the aggregate are needed. There are two well-known end-member cases: homogeneous stress (Sachs, 1928) and homogeneous strain (Taylor-BishopHill (Bishop and Hill, 1951)). The homogeneous-strain model is not well adapted to strongly anisotropic materials, since activation of up to five independent slip systems is necessary to produce arbitrary strain (Castelnau and others, 1996) and the basal plane provides only two independent slip systems. It is also observed in experiments that crystal strain is not homogeneous throughout an ice aggregate (Azuma and Higashi, 1985); this argues against the homogeneous-strain assumption. Experimental data indicate that the homogeneousstress assumption is closer to reality (Azuma, 1995; cf. fig. 4), although interaction with surrounding crystals modifies the deformation to some extent (Azuma, 1995; Castelnau and others, 1996; Sarma and Dawson, 1996; Thorsteinsson, 2000). Incompatibilities arising at grain boundaries, caused by inhomogeneous deformation when using the homogeneousstress assumption, can potentially be relieved by grainboundary migration (Means and Jessell, 1986; Van der Veen and Whillans, 1994; Lliboutry and Duval, 1995). The "true" behavior of ice is somewhere between these two endmember cases, although closer to homogeneous stress. The visco-plastic self-consistent (VPSC) model (Molinari and others, 1987) represents a compromise between the homogeneous-stress and homogeneous-strain bounds (Castelnau and others, 1996). The VPSC is an iterative model. Azuma (1994) calculates the bulk deformation using a "mean orientation" (average $\mathbf{S}$ ) for the crystal aggregate, which effectively assumes that the slip on the basal plane is the same for all crystals. Several other studies have accounted for the anisotropy of ice in an analytical manner. For example, Johnson (1977) modeled deformation of directionally solidified alloys using three parameters, and Lliboutry (1993) and Lliboutry and Duval (1995) used plastic potential theory. There is still no consensus on the exact form that an anisotropic law should take. Results from the Sachs model are comparable with experimental data and other models for strongly anisotropic materials.

Many previous studies have modeled anisotropic deformation of ice including the evolution of fabric (Azuma, 1994, 1995; Van der Veen and Whillans, 1994; Gödert and Hutter, 1998; Morland and Staroszczyk, 1998; Staroszczyk and Gagliardini, 1999). Tracking fabric evolution is an essential part of a complete model for the flow field of an ice sheet, especially in non-steady conditions that lead to major modification of the spatial pattern of fabric. In addition, that approach is required if measured fabric is to be used to infer the history of deformation, stress and temperature. Models that predict the evolution of fabric are necessarily complicated, and often assume linearly viscous behavior or require numerical solutions. These models have not been used to explore the behavior of ice for a range of specified fabrics and stress states, which is the goal of this paper.

\section{FLOW-LAW FORMULATION}

\subsection{Notation}

Vectors (first-rank tensors) are denoted by $\mathbf{v}$ with components $v_{i}$, and second-rank tensors as $\mathbf{A}$ with components $A_{i j}$ where $i, j=1,2,3$. The following notation is used for tensor operations,

$$
\begin{array}{rll}
a=\mathbf{A}: \mathbf{B} & \Leftrightarrow & a=A_{m n} B_{m n}, \\
\mathbf{A}=\mathbf{b} \otimes \mathbf{n} & \Leftrightarrow & A_{i j}=b_{i} n_{j}, \\
\mathbf{v}=\mathbf{A} \cdot \mathbf{n} & \Leftrightarrow & v_{i}=A_{i j} n_{j}, \\
a=\mathbf{n} \cdot \mathbf{k} & \Leftrightarrow & a=n_{i} k_{i} .
\end{array}
$$

\subsection{Single-crystal strain rate}

Ice $\mathrm{I}_{\mathrm{h}}$ deforms almost entirely by dislocation glide on basalplane slip systems. A slip system is defined by a normal vector $\mathbf{n}$ and the slip direction $\mathbf{b}$ (the Burgers vector), where $\mathbf{b}$ is perpendicular to $\mathbf{n}$ (Poirier, 1991). In ice, $\mathbf{n}$ is the crystallographic $c$ axis and $\mathbf{b}$ can be parallel to any of three $a$ axes making $120^{\circ}$ angles to each other. In combination, the slip systems on the basal plane allow glide with equal, or almost equal, ease in any direction on the basal plane (Kamb, 1961). Shear on each slip system is driven by the resolved shear stress (RSS) on the basal plane in the direction of $\mathbf{b}$. To calculate 
the RSS, the first step is to find the traction $\mathbf{T}=\cdot \mathbf{n}$ on the basal plane, where is the Cauchy tensor describing the state of stress acting in the crystal. The traction in the basal plane aligned along $\mathbf{b}$ is $\tau=\mathbf{b} \cdot \mathbf{T}=\mathbf{b} \cdot \mathbf{n}$. This result is described in terms of the Schmid tensor for the slip system defined as

$$
\mathbf{S}=\mathbf{b} \otimes \mathbf{n}
$$

Then the RSS on a given slip system is

$$
\tau=\mathbf{S}:=\mathbf{S}:{ }^{\prime},
$$

where $^{\prime}=+p \mathbf{I}$ with $p=-\sigma_{i i} / 3$. Either the full or the deviatoric part of the stress tensor works, because $\mathbf{S}: \mathbf{I}=\mathbf{n} \cdot \mathbf{b}=0$.

The rate of shearing on a slip system is given by

$$
\frac{\dot{\gamma}}{\dot{\gamma}_{0}}=\left|\frac{\tau}{\tau_{0}}\right|^{n-1} \frac{\tau}{\tau_{0}}
$$

where $\dot{\gamma}_{0}$ and $\tau_{0}$ are the reference shear strain rate and reference resolved stress, respectively, and $n$ gives the power dependence. The velocity gradient tensor $\mathbf{L}^{c}$ for the crystal is then given by

$$
\mathbf{L}^{c}=\sum_{s} \mathbf{S}^{(s)} \dot{\gamma}^{(s)}
$$

where a subscript or superscript in parentheses, $\mathbf{S}^{(s)}$, refers to the slip system $s$ in question, and superscript $c$ refers to a single crystal. The single-crystal strain rate is ${ }^{i c}=\left(\mathbf{L}^{c}+\mathbf{L}^{c T}\right) / 2$, which from Equations (4) and (5) is

$$
\dot{ }^{c} c=\sum_{s} \dot{\gamma}_{0}^{(s)} \mathbf{R}^{(s)}\left|\frac{\mathbf{R}^{(s)}:}{\tau_{0}^{(s)}}\right|^{n-1} \frac{\mathbf{R}^{(s)}:}{\tau_{0}^{(s)}},
$$

where

$$
\mathbf{R}=\frac{\mathbf{S}+\mathbf{S}^{T}}{2}
$$

Since the reference strain rate, $\dot{\gamma}_{0}$, and reference shear stress, $\tau_{0}$, are the same for all three slip systems in the basal plane, one can write $\dot{\gamma}_{0} / \tau_{0}^{n}=\beta \mathcal{A}$, for all the slip systems $(s=1,2,3)$. Here $\beta$ is a constant, and $\mathcal{A}=A(T)$ accounts for the temperature dependence and other isotropic effects; $\mathcal{A}$ is the same function as in Glen's flow law (Paterson, 1994, p. 97). I will assume throughout that $n=3$ (Budd and Jacka, 1989). The strain rate for a single crystal (Equation (6)) is then given by

$$
\dot{"}^{c}=\beta \mathcal{A}\left[\mathbf{R}^{(1)} \tau_{(1)}^{3}+\mathbf{R}^{(2)} \tau_{(2)}^{3}+\mathbf{R}^{(3)} \tau_{(3)}^{3}\right] .
$$

\subsection{Bulk deformation}

With homogeneous-stress assumption, the stress in each crystal is the same as the bulk stress. The velocity gradient of each crystal can be calculated from Equations (3-5). Assuming bulk deformation rate to be the average of the individual crystal deformation rates suggests a modeled velocity gradient of the aggregate

$$
\mathbf{L}^{m}=\frac{1}{N} \sum_{c=1}^{N} \mathbf{L}^{c}
$$

where $N$ is the number of crystals and $\mathbf{L}^{c}$ is the velocity gradient of a single crystal. (This could also be written in terms of volume fractions for non-uniform grain-size.) This modeled velocity gradient $\mathbf{L}^{m}$ does not by itself specify the bulk rotation, since it is derived from the stress tensor. Additional kinematic boundary conditions are required to determine the bulk rotation rate, and the bulk velocity gradient is then defined as $\mathbf{L}=\mathbf{L}^{m}+\dot{\mathbf{\Omega}}^{d}$, where $\dot{\boldsymbol{\Omega}}^{d}$ is the rotation rate necessary to satisfy those boundary conditions (Castelnau and Duval, 1994). When dealing only with strain rates, $\dot{\mathbf{\Omega}}^{d}$ does not have to be considered since it is antisymmetric.

To calculate the bulk properties it is convenient to define a $c$-axis orientation distribution function (ODF) $F(\theta, \phi)$ such that

$$
\iint F(\theta, \phi) \mathrm{d} \theta \mathrm{d} \phi=1
$$

where the $c$-axis orientation in a Cartesian coordinate system is in the form

$$
\mathbf{n}=\sin \theta \cos \phi \hat{\mathbf{x}}_{1}+\sin \theta \sin \phi \hat{\mathbf{x}}_{2}+\cos \theta \hat{\mathbf{x}}_{3},
$$

where $\theta$ is the zenith angle measured from vertical $\hat{\mathbf{x}}_{3}$ and $\phi$ is the azimuth in the external reference frame. The bulk strain rate is then obtained from the integral

$$
\dot{"}=\iint \dot{i n}^{c}(\theta, \phi) F(\theta, \phi) \mathrm{d} \theta \mathrm{d} \phi .
$$

\section{STRAIN RATES UNDER SPEGIFIC STRESS CON- DITIONS FOR GIRDLE FABRICS}

Equation (12) is used to derive analytical equations for the strain rates of anisotropic ice with a girdle fabric subjected to several common stress states. Girdle fabric (Fig. 1) has all zenith angles $\theta$ of the $c$ axes between $\alpha_{0}$ (the girdle angle) and $\alpha$ (the cone angle) with azimuth angles $\phi$ uniformly distributed between $0^{\circ}$ and $360^{\circ}$. The ODF (Equation (10)) for the girdle fabric is

$$
F(\theta, \phi)=\frac{\sin \theta}{2 \pi\left(\cos \alpha_{0}-\cos \alpha\right)}, 0 \leq \alpha_{0} \leq \theta \leq \alpha \leq \frac{\pi}{2} .
$$

$\alpha_{0}=0$ yields the ODF for cone fabric. For isotropic ice $(\alpha=$ $\left.90^{\circ}, \alpha_{0}=0^{\circ}\right)$, the ODF is $F(\theta, \phi)=\sin \theta /(2 \pi)$. A singlemaximum fabric is represented by $\alpha_{0}=0$ and becomes strong as $\alpha$ approaches 0 .

It is convenient to normalize the strain rate with the isotropic response, for the same component, in order to reveal the enhancement relative to isotropic ice. In the past, it has been common to use scalar enhancement factors to describe the effects of anisotropy on the deformation rate. In an anisotropic material the enhancement factor is different for each strain-rate component, and depends on the relative magnitude of the stress components.

\subsection{Uniaxial compression}

In vertical uniaxial compression, the only non-zero component of the Cauchy stress tensor is $\sigma_{33}=\sigma$. The maximum RSS on a basal plane is $\sigma / 2$ at $\theta=45^{\circ}$ (Weertman, 1963), and it is zero at $\theta=0^{\circ}$ and $90^{\circ}$. Examining the velocity gradient tensor of single crystals $\mathbf{L}^{c}$ is useful since the bulk deformation rate is given by an appropriate average of single-crystal deformation rates (Equation (9)). Figure 2 shows the velocity gradient of a single crystal in uniaxial compression depending on its orientation. Each component of the velocity gradient is displayed as a function of zenith $\theta$ and azimuth $\phi$ angles of its $c$-axis $L_{i j}^{c}(\theta, \phi)$, on an equal-area hemispheric plot (see Fig. 1). The components are in a matrix arrangement, with the $L_{11}^{c}$ component in the upper left corner, the $L_{13}^{c}$ component in the upper right corner, and so on. When the bulk fabric has a uniform distribution of $c$ axes within a vertical cone or girdle, the resulting bulk response is obtained by averaging $L_{i j}^{c}(\theta, \phi)$ between the two concentric circles described by $\alpha_{0}$ and $\alpha$. This figure can also be used to see 

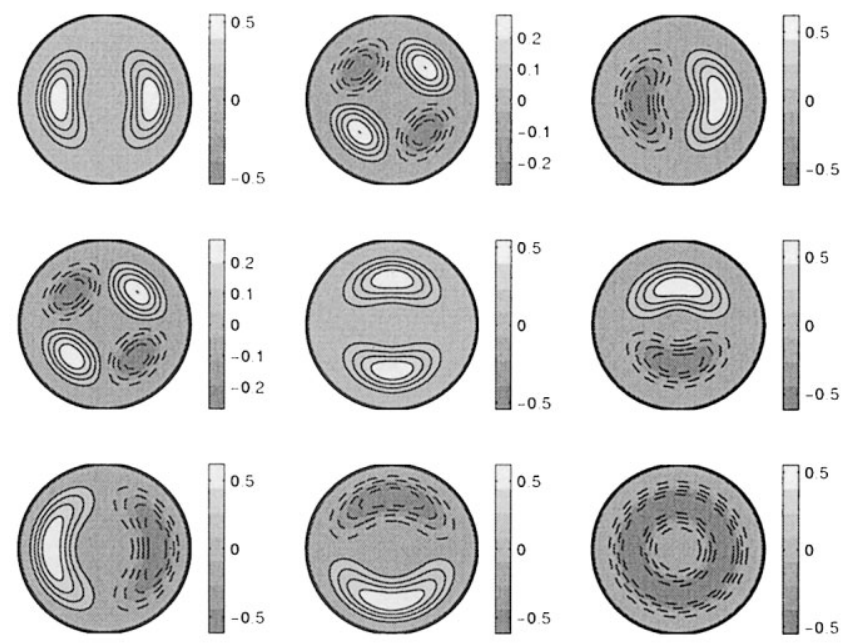

Fig. 2. The velocity gradient tensor, $L_{i j}^{c}(\theta, \phi)$, of a single crystal in uniaxial compression. The crystal is deforming only by glide in the basal plane. Each plot shows a different component $L_{i j}^{c}$ normalized by $\mathcal{A} \sigma^{3}$, with the $L_{11}^{c}$ component in the top left corner and $L_{33}^{c}$ in the bottom right corner. Lighter colors are positive, and darker negative. See Figure 1 for explanation of $\theta$ and $\phi$.

the pattern of strain rate for arbitrary fabric (e.g. a tilted cone or a diamond-maximum pattern) associated with summing the rates for all $c$-axes orientations.

Although all the components of $\mathbf{L}^{c}$ are non-zero in uniaxial compression (Fig. 2) the center-symmetric azimuth averaging will leave only the diagonal terms non-zero. If the symmetry axis of the fabric were tilted to either side there would be a strong shear deformation associated with the compressive stress, for instance.

For a single crystal, the $\dot{\varepsilon}_{33}^{c}=L_{33}^{c}$ component (bottom right in Fig. 2; see equations in Appendix Al) is

$$
\dot{\varepsilon}_{33}^{c}=\beta \mathcal{A} \sigma_{33}^{3} \cos ^{4} \theta \sin ^{4} \theta \frac{1}{72} .
$$

The bulk strain rate for isotropic ice is

$$
\dot{\varepsilon}_{33}=\frac{\beta \mathcal{A} \sigma^{3}}{72 \cdot 2 \pi} \int_{0}^{2 \pi} \int_{0}^{\pi / 2} \cos ^{4} \theta \sin ^{5} \theta \mathrm{d} \theta \mathrm{d} \phi=\frac{\beta A \sigma^{3}}{9} \frac{1}{315} .
$$

It is easy to show that $\dot{\varepsilon}_{11}=\dot{\varepsilon}_{22}=-\frac{1}{2} \dot{\varepsilon}_{33}$, and that all other

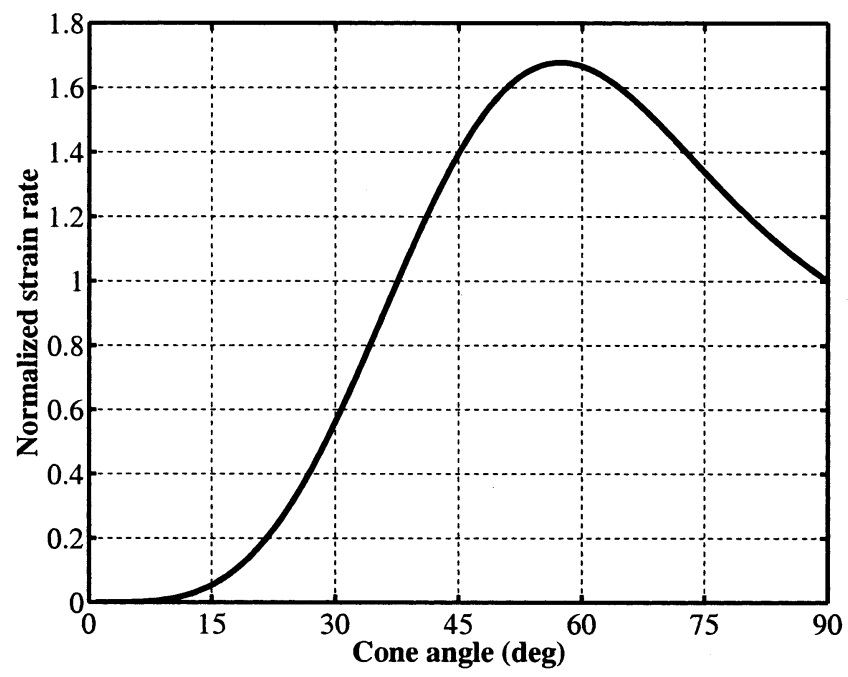

Fig. 3. Normalized axial strain rate $\dot{\varepsilon}_{33}(\alpha) / \dot{\varepsilon}_{33}\left(90^{\circ}\right)$ in uniaxial compression $\sigma_{33}$ as a function of cone angle.
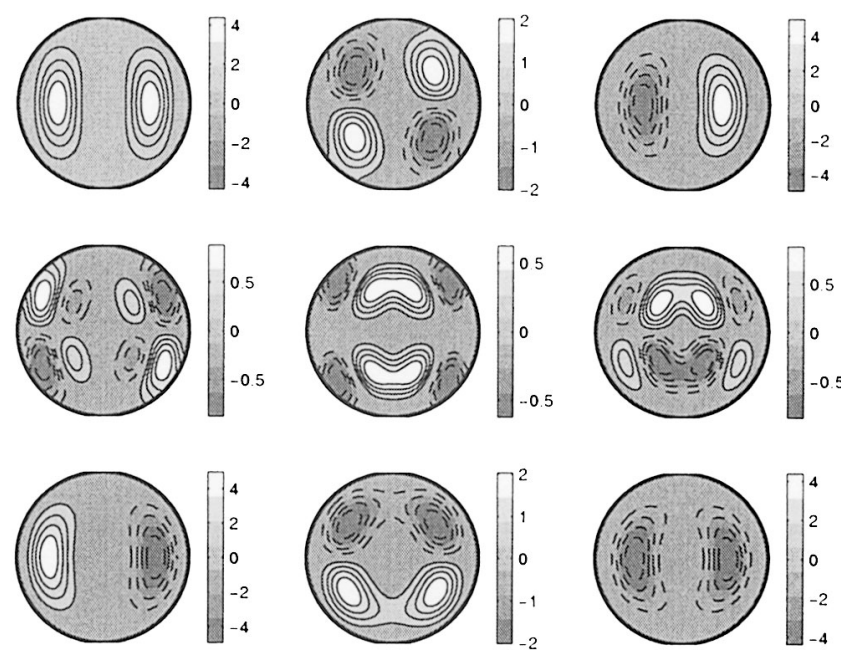

Fig. 4. The velocity gradient tensor, $L_{i j}^{c}(\theta, \phi)$, of a single crystal in pure shear stress $\sigma_{33}=-\sigma_{11}$. The crystal is deforming only by glide in the basal plane. Each plot shows a different component $L_{i j}^{c}$ normalized by $\mathcal{A} \sigma^{3}$, with the $L_{11}^{c}$ component in the top left corner and $L_{33}^{c}$ in the bottom right corner. Lighter colors are positive, and darker negative. See Figure 1 for explanation of $\theta$ and $\phi$.

components are zero. For the same component, Glen's flow law gives $\dot{\varepsilon}_{33}=\frac{2}{9} \sigma^{3} \quad$ (Glen, 1958), which implies $\beta=$ $315 \times 9 \times 2 / 9=630$.

The bulk strain rate $\dot{\varepsilon}_{33}$ for a cone fabric $\left(\alpha_{0}=0\right)$ as a function of cone angle $\alpha$ is calculated from Equations (12) and (14) to be

$$
\begin{gathered}
\dot{\varepsilon}_{33}(\alpha)=\frac{2}{9} \mathcal{A} \sigma^{3} \\
{\left[\frac{\cos ^{5} \alpha\left(35 \sin ^{4} \alpha+20 \sin ^{2} \alpha+8\right)-8}{8(\cos \alpha-1)}\right] .}
\end{gathered}
$$

For a girdle fabric, the bulk strain rate is obtained, in general, using

$$
\dot{\varepsilon}_{i j}\left(\alpha, \alpha_{0}\right)=\frac{\cos \alpha-1}{\cos \alpha-\cos \alpha_{0}}\left(\dot{\varepsilon}_{i j}(\alpha)-\dot{\varepsilon}_{i j}\left(\alpha_{0}\right)\right),
$$

where $\alpha_{0}$ is the girdle angle.

The maximum enhancement in uniaxial compression occurs when all the crystals form a girdle with $\theta=\alpha=\alpha_{0}=$ $45^{\circ}$ (where the RSS is at maximum), then, using Equation (14), $\dot{\varepsilon}_{33}^{c}=\beta \mathcal{A} \sigma^{3} /(16 \times 72)$, and thus the ratio

$$
E_{\mathrm{uc}}^{c}(\max )=\frac{\dot{\varepsilon}_{33}^{\mathrm{c}}\left(\theta=45^{\circ}\right)}{\dot{\varepsilon}_{33}\left(\alpha=90^{\circ}\right)}=\frac{9 \times 315}{16 \times 72}=2.461 .
$$

Figure 3 shows the normalized vertical strain rate, $\dot{\varepsilon}_{33}(\alpha) / \dot{\varepsilon}_{33}\left(90^{\circ}\right)$, for a cone-angle fabric as a function of $\alpha$. Ice in compression is "soft" for all cone angles down to $\alpha \simeq 36^{\circ}$. For smaller cone angles the ice is "stiff". The ice becomes softer as the cone contracts from $\alpha=90^{\circ}$ since many of the "hard" horizontal crystals are being removed. The ice then becomes "hard" when mostly vertical crystals are left (see Fig. 2). The maximum strain rate for cone fabric occurs at $\alpha \simeq 57^{\circ}$ and gives enhancement of 1.678 relative to isotropic ice (Fig. 3).

\subsection{Pure shear}

In pure shear the non-zero components of the stress tensor are $\sigma_{11}=\sigma$ and $\sigma_{33}=-\sigma$. Figure 4 shows the velocity gradient in pure shear for a single crystal. It shows that the $L_{22}^{c}$ component will not average to zero for most cone and girdle 


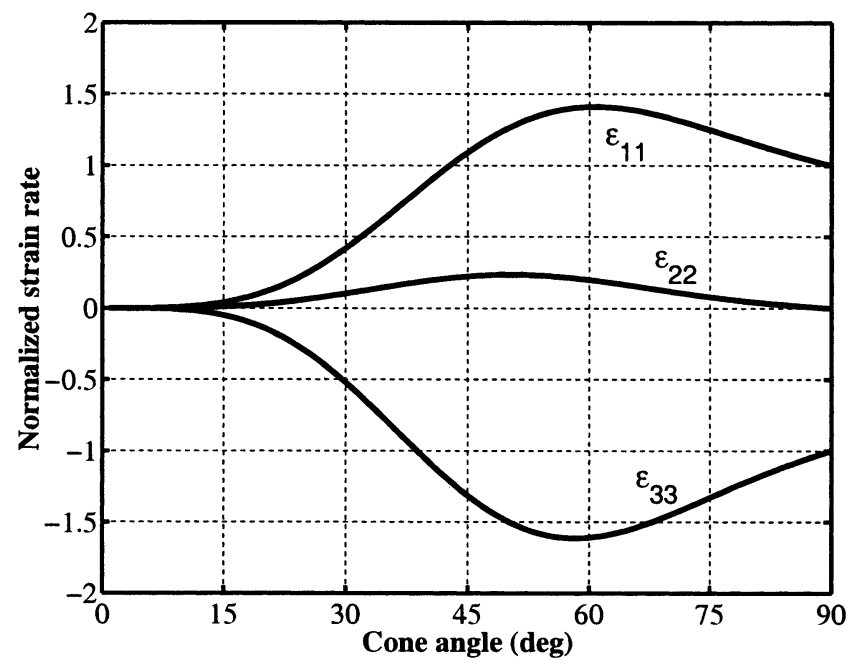

Fig. 5. Normalized strain rate $\dot{\varepsilon}_{i j}(\alpha) /\left(\mathcal{A} \sigma^{3}\right)$ in pure shear stress state $\sigma_{11}=-\sigma_{33}$ as a function of cone angle. The normalized strain rate is equal to the enhancement relative to isotropic ice. Note the non-zero strain rate, $\dot{\varepsilon}_{22}$, which is transverse to the plane of applied stress, and that $\dot{\varepsilon}_{i i}=0$ for all cone angles.

fabrics. Thus, contrary to the isotropic case, there will be an extension normal to the plane of the applied stress. In addition, non-vertically symmetric fabric is likely to produce strong shear $\left(L_{13}^{c}, L_{31}^{c}\right)$ deformation.

The same procedure used to determine bulk strain rates as a function of cone angle in uniaxial compression gives $\beta=630$ and

$$
\begin{aligned}
\dot{\varepsilon}_{11}(\alpha)= & A \sigma^{3}(384090 \cos \alpha+49420 \cos 3 \alpha \\
& -42084 \cos 5 \alpha-2445 \cos 7 \alpha+4235 \cos 9 \alpha \\
& -393216) /[393216(\cos \alpha-1)],
\end{aligned}
$$

$$
\dot{\varepsilon}_{22}(\alpha)=\frac{35 A \sigma^{3}}{6144(1-\cos \alpha)}(177 \cos \alpha+47 \cos 3 \alpha) \sin ^{6} \alpha
$$

$$
\begin{aligned}
\dot{\varepsilon}_{33}(\alpha)= & A \sigma^{3}(16384-15330 \cos \alpha-3080 \cos 3 \alpha \\
& +2016 \cos 5 \alpha+255 \cos 7 \alpha-245 \cos 9 \alpha) \\
& /[16384(\cos \alpha-1)] .
\end{aligned}
$$

Figure 5 shows the non-zero components of the strain rate tensor as a function of cone angle, normalized by $A \sigma^{3}$, which in this case gives the enhancement relative to isotropic ice (for $\dot{\varepsilon}_{11}$ and $\dot{\varepsilon}_{33}$ ). Note in particular that, although relatively small, the $\dot{\varepsilon}_{22}$ component is non-zero for a range of cone angles (see Fig. 4). This means that a block of ice deforming in a pure shear-stress state will expand/contract in a direction perpendicular to the plane defined by the applied stress. Correspondingly, a stress $\sigma_{22}$ would be required to impose plane strain rate $\dot{\varepsilon}_{11}=-\dot{\varepsilon}_{33}, \dot{\varepsilon}_{22}=0$.

\subsection{Simple shear}

In simple shear-stress state, the only non-zero components of the stress tensor are $\sigma_{31}=\sigma_{13}=\sigma$. The maximum RSS on the basal plane is $\sigma$ for zenith angles of $\theta=0^{\circ}$ and $90^{\circ}$, and it is zero at $45^{\circ}$, in the plane of applied stress. Figure 6 shows that the $L_{13}^{c}$ term (top right) is large for nearly vertical crys-
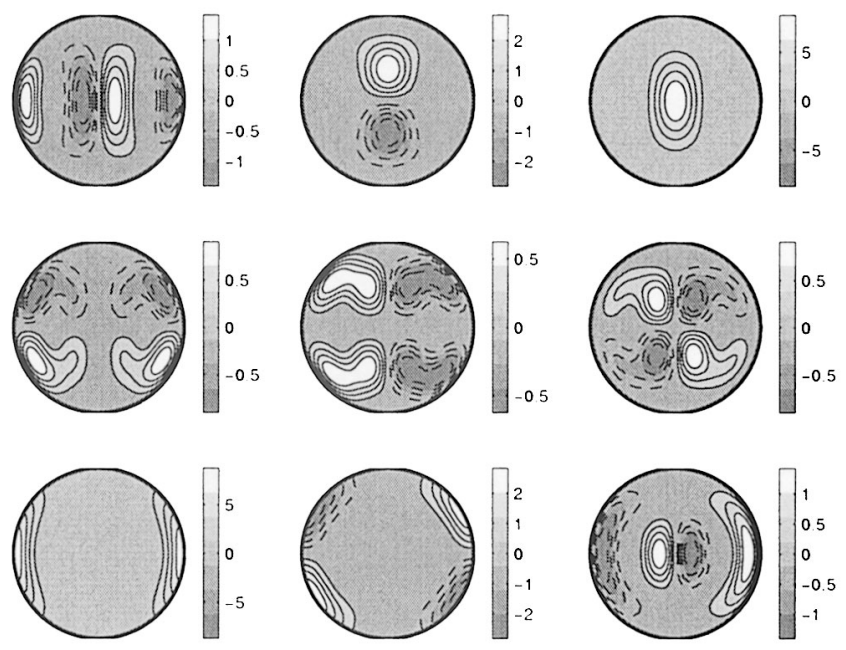

Fig. 6. The velocity gradient tensor, $L_{i j}^{c}(\theta, \phi)$, of a single crystal in simple shear stress $\sigma_{13}=\sigma_{31}$. The crystal is deforming only by glide in the basal plane. Each plot shows a different component $L_{i j}^{c}$ normalized by $\mathcal{A} \sigma^{3}$, with the $L_{11}^{c}$ component in the top left corner and $L_{33}^{c}$ in the bottom right corner. Lighter colors are positive, and darker negative. See Figure 1 for explanation of $\theta$ and $\phi$.

tals (small zenith angle) and the $L_{31}^{c}$ term (bottom left) is large for nearly horizontal crystals (large zenith angle). For isotropic ice, these two terms contribute equally to the aggregate deformation. Therefore, there is no bulk rotation, which demonstrates the need to define the rotation rate $\dot{\boldsymbol{\Omega}}^{d}$ to satisfy kinematic boundary conditions. Also note that, although smaller, several other velocity gradient components are nonzero. They average to zero for the vertical cone distribution of $c$ axes used here, but for other distributions they might not. For a single crystal, the shear strain rate (Equation (6)) is

$$
\begin{aligned}
& \dot{\varepsilon}_{13}^{c}(\theta, \phi)=\beta A \sigma_{13}^{3} \frac{1}{162}\left(\left[\cos \phi\left(\cos ^{2} \theta-\sin ^{2} \theta\right)\right]^{4}\right. \\
& +\frac{1}{16}\left\{\left[\sin ^{2} \theta \cos \phi \cos \theta(\cos \theta \cos \phi+\sqrt{3} \sin \phi)\right]^{4}\right. \\
& \left.\left.+\left[\sin ^{2} \theta \cos \phi-\cos \theta(\cos \theta \cos \phi-\sqrt{3} \sin \phi)\right]^{4}\right\}\right) .
\end{aligned}
$$

For an isotropic distribution,

$$
\dot{\varepsilon}_{13}=\frac{1}{2 \pi} \int_{0}^{2 \pi} \int_{0}^{\pi / 2} \dot{\varepsilon}_{13}^{c} \sin \theta \mathrm{d} \theta \mathrm{d} \phi=\beta A \sigma^{3} \frac{1}{630},
$$

Glen's flow law gives $\dot{\varepsilon}_{13}=A \sigma^{3}$, so as before, $\beta=630$. The bulk strain rate for cone fabric is

$$
\begin{aligned}
& \dot{\varepsilon}_{13}(\alpha)=A \sigma^{3}(2730 \cos \alpha-35 \cos 3 \alpha+357 \cos 5 \alpha \\
& -15 \cos 7 \alpha+35 \cos 9 \alpha-3072) /[3072(\cos \alpha-1)] .
\end{aligned}
$$

The maximum enhancement in simple shear occurs for a perfect vertical alignment of all the crystals

$$
E_{\mathrm{SS}}(\max )=\frac{\dot{\varepsilon}_{13}\left(\alpha=\theta=0^{\circ}\right)}{\dot{\varepsilon}_{13}\left(\alpha=90^{\circ}\right)}=\frac{1 / 144}{1 / 630}=\frac{35}{8}=4.375 .
$$

Figure 7 shows the shear strain rate, normalized by the isotropic shear strain rate, as a function of cone angle. Note that for cone angles of $60-90^{\circ}$ the deformation is actually a little slower than for isotropic ice $(E<1)$, since many of the 


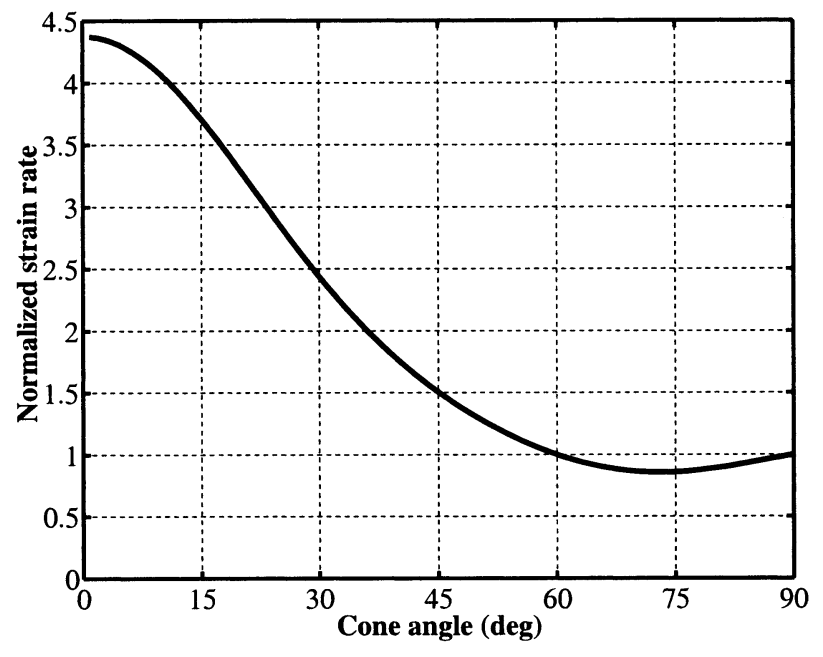

Fig. 7. Normalized strain rate $\dot{\varepsilon}_{13}(\alpha) / \dot{\varepsilon}_{13}\left(90^{\circ}\right)$ in simple shear as a function of cone angle. Note that the enhancement is smaller than 1 for cone angles of 60-90 ${ }^{\circ}$, with a minimum of 0.857 at a cone angle $\alpha=73^{\circ}$.

easily deformable horizontal crystals are absent (see Fig. 6, bottom left).

\subsection{Combined uniaxial compression and simple shear}

Particularly relevant states of stress for ice sheets are simple shear driving the main outward flow of ice and secondary stress associated with vertical motions accommodating accumulation.

The combination of uniaxial compression and simple shear stress exists in ice sheets in the vicinity of ice domes, for example. In this case the stress tensor has the form

$$
\sigma_{i j}=\left[\begin{array}{ccc}
0 & 0 & \tau \\
0 & 0 & 0 \\
\tau & 0 & \sigma
\end{array}\right] .
$$

An additional mean compression does not change the deformation. The RSS on a crystal slip system is

$$
\tau_{(s)}=\underbrace{\left(S_{13}+S_{31}\right) \tau}_{\mathcal{T}_{13}}+\underbrace{S_{33} \sigma}_{\mathcal{T}_{33}},
$$

which, with $n=3$, becomes, $\tau_{(s)}^{3}=\mathcal{T}_{13}^{3}+3 \mathcal{T}_{13}^{2} \mathcal{I}_{33}+$ $3 \mathcal{T}_{13} \mathcal{T}_{33}^{2}+\mathcal{T}_{33}^{3}$. The first and the last term are exactly the values of $\tau_{(s)}$ for simple shear and uniaxial compression acting alone. The equations for the non-zero velocity gradient components $\left(L_{11}, L_{22}, L_{33}, L_{13}\right)$ as a function of stress and cone angle are given in Appendix A2.

Figures 8 and 9 show the bulk vertical strain rate $\left(\dot{\varepsilon}_{33}\right)$ and shear strain rate $\left(\dot{\varepsilon}_{13}\right)$ as a function of vertical cone angle. For the $\dot{\varepsilon}_{33}$ component, the total strain rate is the sum of two terms (Fig. 8), as for isotropic ice, but each depends on the fabric $\dot{\varepsilon}_{33}(\alpha)=\left(f(\alpha) \sigma^{2}+g(\alpha) \tau^{2}\right) \sigma$. (See Appendix A2.) The $\dot{\varepsilon}_{13}$ component (Fig. 9) has a similar form $\dot{\varepsilon}_{13}(\alpha)=\left(h(\alpha) \sigma^{2}+j(\alpha) \tau^{2}\right) \tau$. For isotropic ice, the contribution from each term, and the sum, is exactly the same as in Glen's flow law.

Figure 10 shows the normalized bulk response $\dot{\varepsilon}_{33}(\alpha, \tau / \sigma) / \dot{\varepsilon}_{33}\left(90^{\circ}, \tau / \sigma\right)$ as a function of cone angle and the ratio of shear to compressive stress. Figure 11 shows the $\dot{\varepsilon}_{13}(\alpha, \tau / \sigma) / \dot{\varepsilon}_{13}\left(90^{\circ}, \tau / \sigma\right)$ component. Note how the peak enhancement in vertical strain rate increases with increasing shear stress, and moves from $\alpha \simeq 60^{\circ}$ for $\tau / \sigma<1$ to $\alpha \simeq 35^{\circ}$ for $\tau / \sigma>1$. Also, note the rapid variation of verti-

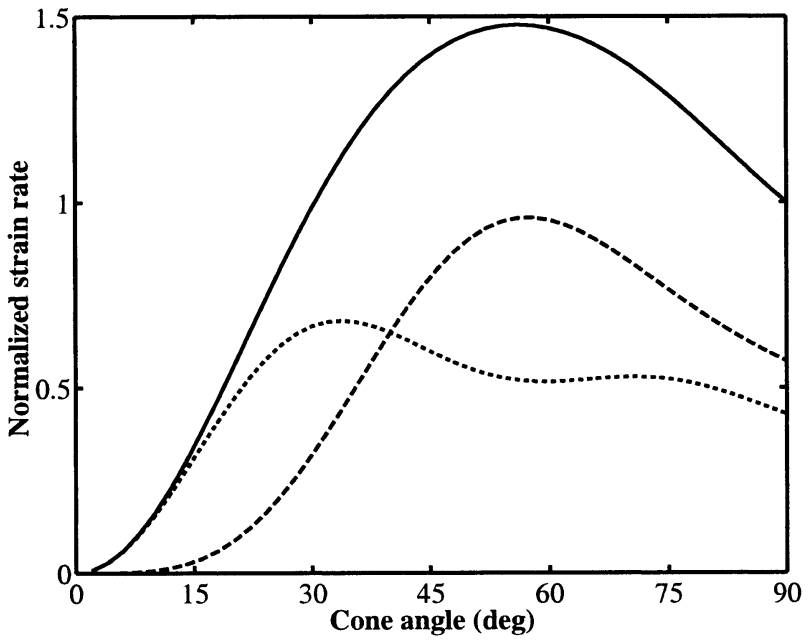

Fig. 8. Normalized strain rate, $\dot{\varepsilon}_{33}(\alpha) / \dot{\varepsilon}_{33}\left(90^{\circ}\right)$ (solid line) as a function of cone angle in combined compression and shear. The resulting strain rate is made up of two terms, one that depends on $\sigma^{3}$ only (dashed line), and one that depends on the shear stress and compression $\tau^{2} \sigma$ (dotted line). Here $\tau / \sigma=0.5$.

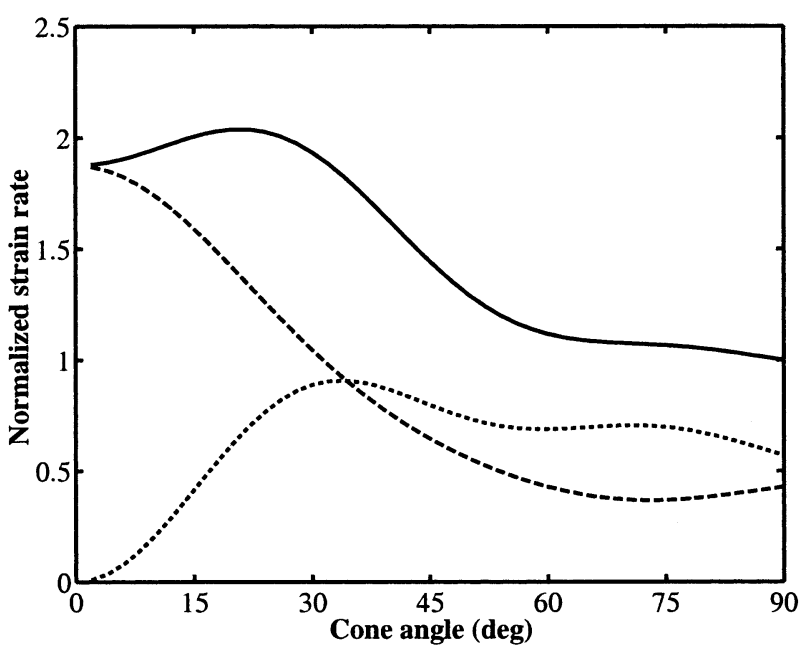

Fig. 9. Normalized strain rate $\dot{\varepsilon}_{13}(\alpha) / \dot{\varepsilon}_{13}\left(90^{\circ}\right)$ (solid line) as a function of cone angle in combined compression and shear. The resulting strain rate is made up of two terms, one due to the shear stress $\tau^{3}$ (dashed line), and one caused by the uniaxial compression stress and shear $\sigma^{2} \tau$ (dotted line). Here $\tau / \sigma=0.5$.

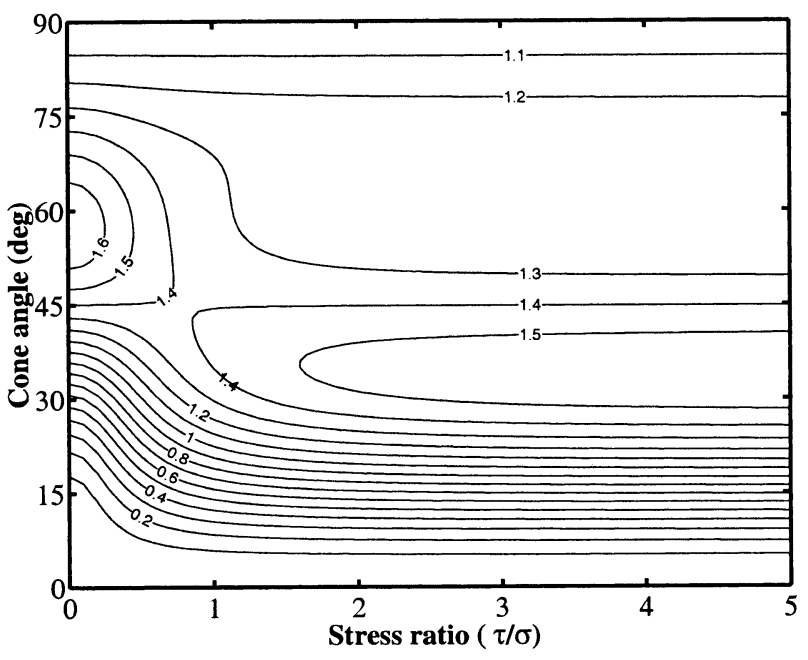

Fig. 10. The normalized vertical strain rate, $\dot{\varepsilon}_{33}(\alpha, \tau / \sigma) / \dot{\varepsilon}_{33}$ $\left(\alpha=90^{\circ}, \tau / \sigma\right)$, as a function of the ratio of shear to compressive stress, $\tau / \sigma$, and cone angle, $\alpha$. Note that the compression has a maximum rate at cone angle about $60^{\circ}$ when $\tau / \sigma$ is small and near $35^{\circ}$ at larger ratios. 


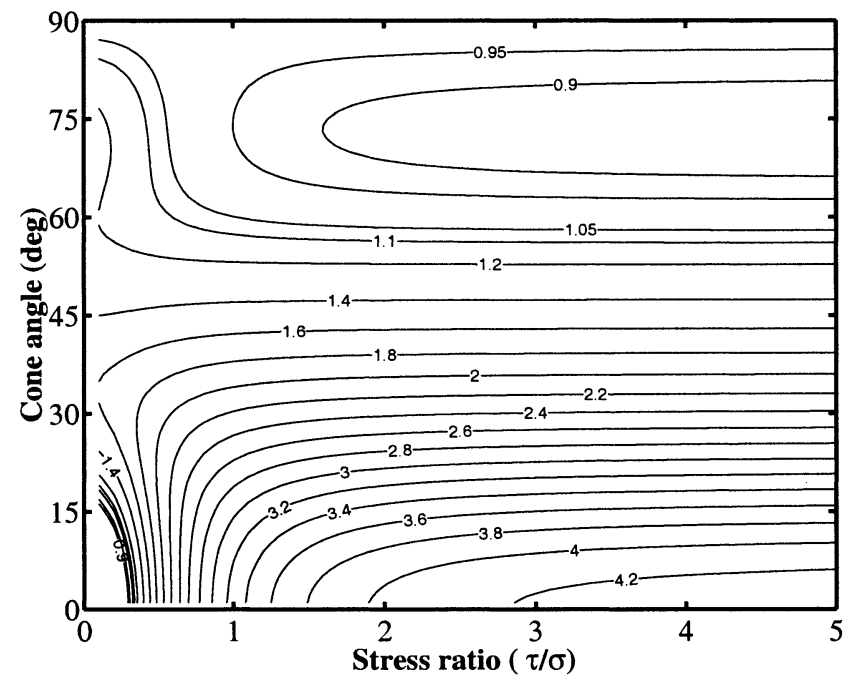

Fig. 11. The normalized shear strain rate, $\dot{\varepsilon}_{13}(\alpha, \tau / \sigma) / \dot{\varepsilon}_{13}$ $\left(\alpha=90^{\circ}, \tau / \sigma\right)$, as a function of the ratio of shear to compressive stress, $\tau / \sigma$, and cone angle, $\alpha$.

cal strain rate at small cone angles $\alpha$, especially with increasing shear stress. The shear strain rate has a simpler pattern, except when $\tau \sim \sigma$, where the enhancement has a fairly complex structure at small cone angles $\alpha$.

Combined pure and simple shear stress, for cone fabric, gives nearly identical results. The enhancement shown in Figures 10 and 11 can also result from tilting a cone in pure shear stress $(\tau=0)$, since a simple shear stress state can be represented as rotated pure shear stress. The tilt angle of the cone normal $\xi$ is given by $\xi=\arctan (\tau / \sigma) / 2$. This demonstrates that even in a pure shear stress state (or uniaxial compression), at an ice divide for instance, a tilted cone (symmetry axis not vertical) will result in a non-zero shear strain rate $\left(\dot{\varepsilon}_{13}\right)$.

\section{DISGUSSION}

Several models, of various levels of complexity, have been used to account for the effects of anisotropy. The simplest model is one scalar enhancement factor. That works when relating a single component of stress to corresponding strain rate in a simple state of stress, but cannot be used for more complex stress states. An "enhancement factor", as a function of cone angle, depends on the strain-rate component and the relative magnitude of stress components (see Fig. 9). A model described byJohnson (1977) uses three parameters $(\lambda, \mu, \nu)$ to characterize vertically symmetric transversely isotropic anisotropy. The equations for the strain rates according to Johnson's model are in Appendix A3. The parameters are defined from three deformation experiments: uniaxial compression along the $\hat{\mathbf{x}}_{3}$ axis gives $\lambda^{2}=\dot{\varepsilon}_{33} / \sigma_{33}^{3}$, compression along the $\hat{\mathbf{x}}_{1}$ axis gives $\mu^{2}=\dot{\varepsilon}_{11} / \sigma_{11}^{3}$ and simple shear deformation gives $\nu^{2}=2 \dot{\varepsilon}_{13} / \sigma_{13}^{3}$. Their values can be derived as functions of cone angle (Appendix A3).

It is interesting to compare the strain rate calculated using the Johnson model to the strain rate calculated with the model developed here. Figures 12 and 13 show a comparison of the $\dot{\varepsilon}_{33}$ and $\dot{\varepsilon}_{13}$ strain-rate components, respectively, calculated using the Sachs and Johnson models in combined pure and simple shear stress. For the $\dot{\varepsilon}_{33}$ component the two profiles look similar for small stress ratio $\tau / \sigma$, but the differences become more obvious as the ratio (shear stress) increases.

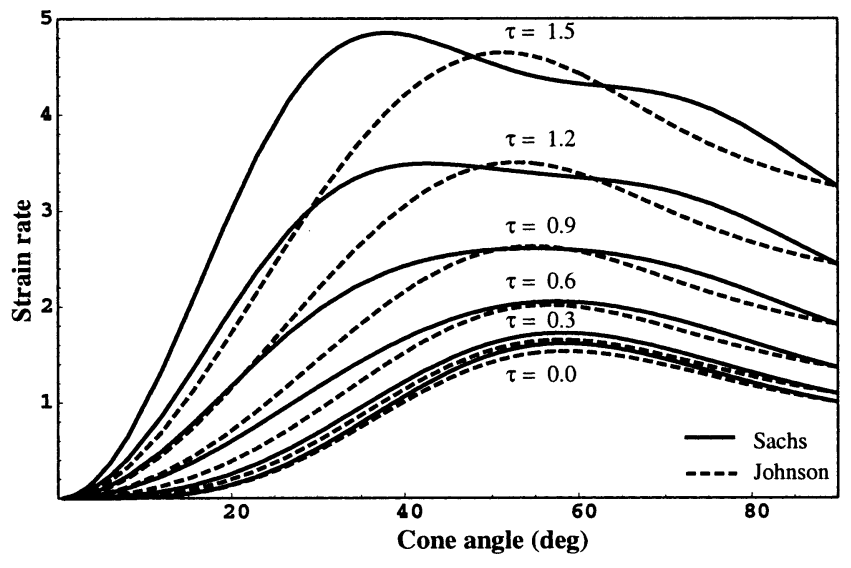

Fig. 12. Comparing $\dot{\varepsilon}_{33}(\alpha)$ obtained using the Sachs and Johnson formulation for combined pure shear $\left(\sigma_{11}=-\sigma_{33}=-1\right)$ and simple shear $\tau=\sigma_{13}=(0,0.3,0.6,0.9,1.2,1.5)$.

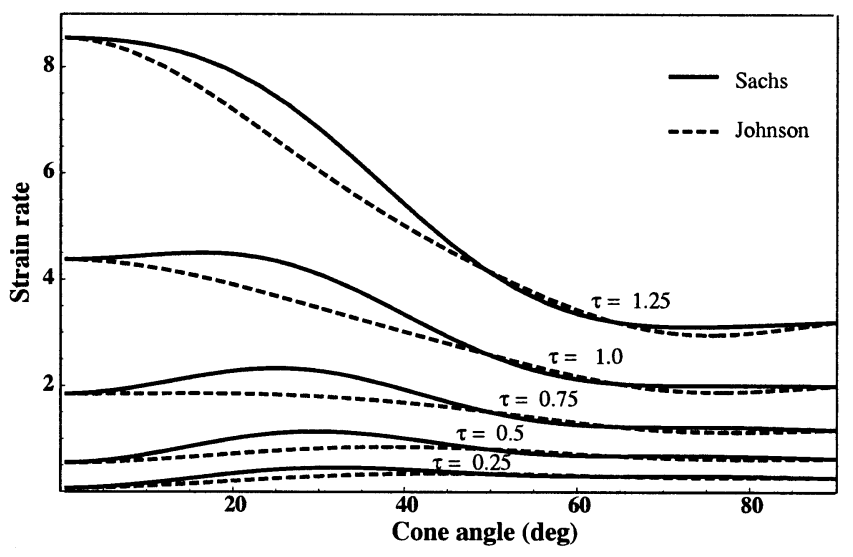

Fig. 13. Comparing $\dot{\varepsilon}_{13}(\alpha)$ obtained using the Sachs and Johnson formulation for combined pure shear $\left(\sigma_{11}=-\sigma_{33}=-1\right)$ and simple shear $\tau=\sigma_{13}=(0.25,0.5,0.75,1.0,1.25)$.

Three parameters cannot simulate the effects of anisotropy completely (five to seven are needed, as noted by Lliboutry (1993) and Lliboutry and Duval (1995)). The Johnson model captures the major effects of the anisotropy, but not all the details. Calculating the strain rate using Equation (12) becomes laborious as the number of non-zero stress components increases. The Johnson model depends only on the three parameters regardless of the stress pattern, and therefore offers an efficient way to account for the major effects of anisotropy for highly complicated stress states.

The formulation presented here gives a maximum enhancement of 4.375 in simple shear. Laboratory experiments often indicate a value close to 9 (Budd and Jacka, 1989), although several other factors besides fabric anisotropy may influence the experimental results. Some formulations of anisotropy, such as Azuma's model (Azuma, 1994, 1995; Azuma and Goto-Azuma, 1996), the VPSC model (Castelnau and others, 1996) and the Thorsteinsson (2000) model with nearest-neighbor interaction, produce enhancement in simple shear that is close to 9. However, it is important to note that the style of deformation is very similar for all those models.

I model the deformation of ice considering only slip in the basal plane. Other slip systems are unlikely to contribute significantly to the deformation of ice, except in very special cases. One such case is clearly demonstrated in Figure 3, for uniaxial compression, at small cone angles $\left(\alpha<10^{\circ}\right)$. Here $E \rightarrow 0$ as $\alpha \rightarrow 0$. Other slip systems, probably the pyramidal and prismatic, will determine the correct deformation 

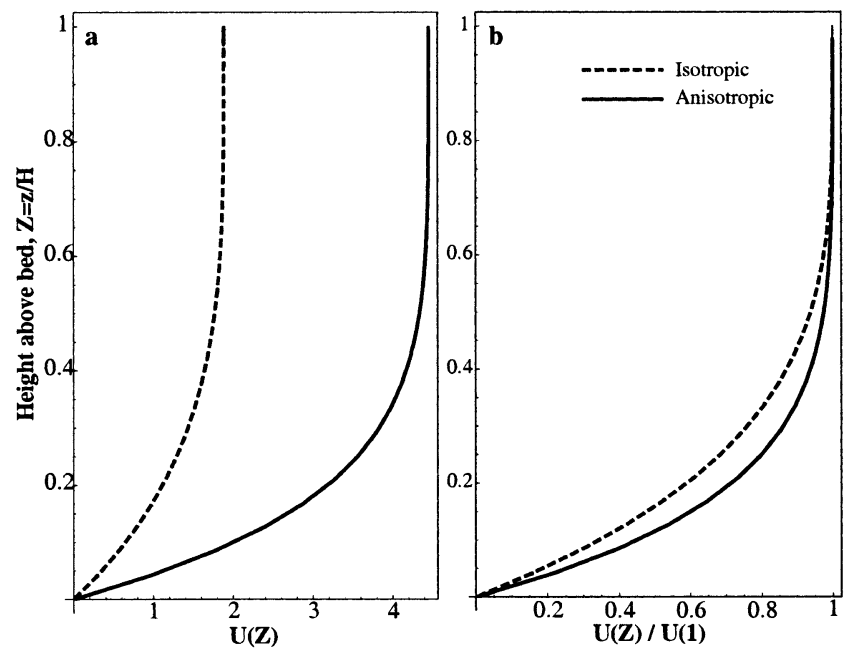

Fig. 14. Horizontal velocity $U(Z)$ as a function of height above bed $(Z=z / H$, where $H$ is the ice thickness $)$ for isotropic ice $\left(\alpha(Z)=90^{\circ}\right.$; dashed line $)$ and anisotropic ice $\left(\alpha(Z)=20^{\circ}\right.$ $+70^{\circ} \mathrm{Z}$; solid line). The anisotropy enhances the deformation $(a)$, and concentrates it closer to the bed $(b)$.

rate as $\alpha \rightarrow 0$. This value is likely small in any case, since these other slip systems are at least an order of magnitude stiffer than slip systems in the basal plane.

At most locations in an ice sheet the vertical strain rate remains constant from the surface down to some depth close to the bed (e.g. Dansgaard and Johnsen, 1969). For isothermal, isotropic ice, the vertical deviatoric stress would also be constant down to that depth (changes associated with temperature are neglected here since the effect is the same for isotropic and anisotropic ice). However, when anisotropy is taken into account, the deviatoric stress must vary with depth to maintain a constant strain rate, since the fabric varies with depth (Fig. 3). If the strain rate is constant, and the deviatoric vertical compressive stress near the surface $\left(\alpha \simeq 90^{\circ}\right)$ is $\sigma=\sigma_{0}$, then the deviatoric stress where the ice is softest $\left(\alpha=57^{\circ}\right)$ is reduced to $0.84 \sigma_{0}$, and where it is stiff it is increased (e.g. $\sigma\left(\alpha=20^{\circ}\right)=1.875 \sigma_{0}$, and $\sigma\left(\alpha=5^{\circ}\right)=11 \sigma_{0}$ ). The anisotropy thus causes stress redistribution, concentrating stress on the stiff sections $\left(\alpha<36^{\circ}\right)$ and reducing it on the soft sections $\left(\alpha>36^{\circ}\right)$. Any asymmetry in the fabric of stiff layers can therefore easily lead to anomalous deformation.

The typical vertical symmetry of fabric implies alteration of the velocity profile in a parallel-sided slab model. In a parallel-sided slab model only the slope-parallel velocity is non-zero, and it changes only with depth according to $\mathrm{d} u / \mathrm{d} z=2 \dot{\varepsilon}_{13}$; glaciologists sometimes call this "laminar flow" (Paterson, 1994, p. 251). Figure 14 shows velocity profiles for isotropic and anisotropic ice. Here the cone angle for the anisotropic ice is assumed to change linearly from $90^{\circ}$ at the surface to $20^{\circ}$ at the bottom, while the stress and temperature distributions are the same for both the isotropic and anisotropic ice. Due to the anisotropy, the surface velocity is 2.36 times larger than in the isotropic case, and the mean velocity is 2.5 times larger. For isotropic ice, the ratio of the mean velocity to the surface velocity $\bar{u} / u_{\mathrm{s}}$ is 0.8 $\left(\bar{u} / u_{\mathrm{s}}=(n+1) /(n+2) ; n=3\right)$. This ratio $\left(\bar{u} / u_{\mathrm{s}}\right)$ is 0.848 in the anisotropic case examined here. It will in general be different from 0.8; the magnitude of the difference depends on the variation of the cone angle with depth.

Even though I used the combination of uniaxial compression and simple shear to demonstrate how the anisotropy affects the vertical and shear strain rates, the same conclu- sions apply for a combined pure and simple shear stress state for the vertically symmetric fabric used here. In pure shear deformation, the fabric is unlikely to maintain azimuthal symmetry (Thorsteinsson, 2000). Figure 10 shows that the vertical strain rate in ice at an ice divide (where there is no simple shear stress) will change significantly should the ice divide subsequently migrate (adding shear stress). If there are patches of ice with a non-vertical symmetry axis at a divide, they will be subject to shear, so there is a potential for folding (Azuma and Goto-Azuma, 1996; Mangeney and others, 1997; Thorsteinsson and Waddington, in press).

\section{CONCLUSIONS}

It is clear that non-isotropic fabric can lead to very complex deformation patterns. The strain-rate tensor does not always have the same non-zero components as the applied stress tensor, even for a highly symmetric vertical girdle fabric. This will generally occur when the stress and fabric symmetries differ, as in the case of pure shear (Fig. 5). When pure shear stress is applied to a block of ice with a girdle fabric, the ice has a nonzero deformation normal to the plane of applied stress. In a simple shear-stress state, there is a strain-rate enhancement when $\alpha<60^{\circ}$, but slight de-enhancement for $60^{\circ}<\alpha<90^{\circ}$. The enhancement accounts for much of the excess bed-parallel shear deformation at depths in ice sheets where cone-like fabrics are well developed (Thorsteinsson and others, 1999). In uniaxial compression, ice with cone angles $36^{\circ}<\alpha<90^{\circ}$ is soft relative to isotropic ice, with a maximum at $\alpha=57^{\circ}$.

Using a single enhancement function leads to erroneous strain rates in combined stress states. This is because each of the non-zero stress components contributing to the deformation is weighted with a function that depends on the fabric, unlike the case of isotropic ice. The axial strain rate in combined uniaxial compression and simple shear stress state, for example, is $\dot{\varepsilon} \propto\left(f \tau^{2}+g \sigma^{2}\right) \sigma$, where $f, g$ are functions of fabric. The formulation presented here captures the essential character of deformation of strongly anisotropic ice, and represents a major improvement over the use of scalar enhancement factors to account for anisotropic effects, while remaining relatively simple.

\section{ACKNOWLEDGEMENTS}

I thank E. D. Waddington, C. F. Raymond and T. Neumann for valuable comments on the manuscript at various stages, and also J.W. Glen (scientific editor), W. D. Harrison (chief editor) and two anonymous reviewers. This work was supported by the U.S. National Science Foundation, under grant Nos. OPP-9123660, OPP-9526707 and OPP-9815136.

\section{REFERENGES}

Alley, R. B. 1992. Flow-law hypotheses for ice-sheet modeling. F. Glaciol., 38(129), 245-256.

Alley, R. B., A. J. Gow and D. A. Meese. 1995. Mapping $c$-axis fabrics to study physical processes in ice. F. Glaciol., 41 (137), 197-203.

Anandakrishnan, S., J.J. FitzPatrick, R. B. Alley, A. J. Gow and D. A. Meese. 1994. Shear-wave detection of asymmetric $c$-axis fabrics in the GISP2 ice core, Greenland. F. Glaciol., 40(136), 491-496.

Azuma, N. 1994. A flow law for anisotropic ice and its application to ice sheets. Earth Planet. Sci. Lett., 128(3-4), 601-614.

Azuma, N. 1995. A flow law for anisotropic polycrystalline ice under uniaxial compressive deformation. Cold Reg. Sci. Technol., 23(2), 137-147.

Azuma, N. and K. Goto-Azuma. 1996. An anisotropic flow law for ice-sheet ice and its implications. Ann. Glaciol., 23, 202-208. 
Azuma, N. and A. Higashi. 1985. Formation processes of ice fabric pattern in ice sheets. Ann. Glaciol., 6, 130-134.

Bishop, J. F.W. and R. Hill. 1951. A theory of the plastic distortion of a polycrystalline aggregate under combined stresses. Philos. Mag., 7th Ser., 42, 414-427.

Budd, W. F. and T. H. Jacka. 1989. A review of ice rheology for ice sheet modelling. Cold Reg. Sci. Technol., 16(2), 107-144.

Castelnau, O. and P. Duval. 1994. Simulations of anisotropy and fabric development in polar ices. Ann. Glaciol., 20, 277-282.

Castelnau, O., P. Duval, R. Lebensohn and G. R. Canova. 1996. Viscoplastic modeling of texture development in polycrystalline ice with a self-consistent approach: comparison with bound estimates. F. Geophys. Res., 101 (B6), 13,851-13,868.

Cuffey, K. M., T. Thorsteinsson and E. D. Waddington. 2000. A renewed argument for crystal size control of ice sheet strain rates. 7. Geophys. Res., 105(B12), 27,889-27,894.

Dahl-Jensen, D. and N. S. Gundestrup. 1987. Constitutive properties of ice at Dye 3, Greenland. International Association of Hydrological Sciences Publication 170 (Symposium at Vancouver 1987 - The Physical Basis of Ice Sheet Modelling), 31-43.

Dansgaard, W. and S. J. Johnsen. 1969. A flow model and a time scale for the ice core from Camp Century, Greenland. F. Glaciol., 8(53), 215-223.

Duval, P. 1981. Creep and fabrics of polycrystalline ice under shear and compression. 7. Glaciol., 27(95), 129-140.

Duval, P. and O. Castelnau. 1995. Dynamic recrystallization of ice in polar ice sheets. 7. Phys. (Paris), IV (5), Colloq. C3, 197-205. (Supplément au 3.)

Duval, P. and H. le Gac. 1982. Mechanical behaviour of Antarctic ice. Ann. Glaciol., 3, 92-95.

Duval, P., M. F. Ashby and I. Anderman. 1983. Rate-controlling processes in the creep of polycrystalline ice. F. Phys. Chem., 87(21), 4066-4074.

Glen, J.W. 1958. The flow law of ice: a discussion of the assumptions made in glacier theory, their experimental foundation and consequences. International Association of Scientific Hydrology Publication 47 (Symposium at Chamonix 1958 - Physics of the Movement of the Ice), 171-183.

Gödert, G. and K. Hutter. 1998. Induced anisotropy in large ice shields: theory and its homogenization. Continuum Mech. Thermodyn., 10(5), 293-318.

Gow, A. J. and 6 others. 1997. Physical and structural properties of the Greenland Ice Sheet Project 2 ice cores: a review. f. Geophys. Res., 102(C12), 26,559-26,575.

Gundestrup, N.S. and B. L. Hansen. 1984. Bore-hole survey at Dye 3, south Greenland. 7. Glaciol., 30(106), 282-288.

Herron, S. L., C. C. Langway, Jr and K. A. Brugger. 1985. Ultrasonic velocities and crystalline anisotropy in the ice core from Dye 3, Greenland. In Langway, C. C., Jr, H. Oeschger and W. Dansgaard, eds. Greenland ice core: geophysics, geochemistry, and the environment. Washington, DC, American Geophysical Union, 23-31. (Geophysical Monograph 33. )

Johnson, A. F. 1977. Creep characterization of transversely-isotropic metallic material. 7. Mech. Phys. Solids, 25, 117-126.

Kamb, W. B. 1961. The glide direction in ice. F. Glaciol., 3(30), 1097-1106.

Kohnen, H. and A. J. Gow. 1979. Ultrasonic velocity investigations of crystal anisotropy in deep ice cores from Antarctica. CRREL Rep. 79-10.

Lliboutry, L. 1993. Anisotropic, transversely isotropic nonlinear viscosity of rock ice and rheological parameters inferred from homogenization. Int. 7. Plasticity, 9(5), 619-632.

Lliboutry, L. and P. Duval. 1985. Various isotropic and anisotropic ices found in glaciers and polar ice caps and their corresponding rheologies. Ann. Geophysicae, 3(2), 207-224.

Mangeney, A., F. Califano and K. Hutter. 1997. A numerical study of anisotropic, low Reynolds number, free surface flow for ice sheet modeling. F. Geophys. Res., 102(B10), 22,749-22,764.

Means, W. D. and M.W. Jessell. 1986. Accommodation migration of grain boundaries. Tectonophysics, 127(1-2), 67-86.

Molinari, A., G. Canova and S. Ahzi. 1987. A self consistent approach of the large deformation polycrystal viscoplasticity. Acta Metall., 35(12), 2983-2994.

Morland, L. and R. Staroszczyk. 1998. Viscous response of polar ice with evolving fabric. Continuum Mech. Thermodyn., 10(3), 135-152.

Paterson, W. S. B. 1991. Why ice-age ice is sometimes "soft". Cold Reg. Sci. Technol., 20 (1), 75-98.

Paterson, W. S. B. 1994. The physics of glaciers. Third edition. Oxford, etc., Elsevier. Poirier, J.-P. 1991. Introduction to the physics of the Earth's interior. In Putnis, A. and R. C. Lieberman, eds. Cambridge topics in mineral physics and chemistry. Cambridge, Cambridge University Press, 154-157.

Russell-Head, D. S. and W. F. Budd. 1979. Ice-sheet flow properties derived from bore-hole shear measurements combined with ice-core studies. $\mathcal{F}$. Glaciol., 24(90), 117-130.

Sachs, G. 1928. Zur Ableitung einer Fließbedingung. Z. Vereins Dtsch. Ing., 72(8), 734-736

Sarma, G. B. and P. R. Dawson. 1996. Texture predictions using a polycrystal plasticity model incorporating neighbor interactions. Int. 7. Plasticity, 12(8), 1023-1054
Staroszczyk, R. and O. Gagliardini. 1999. Two orthotropic models for strain-induced anisotropy of polar ice. f. Glaciol., 45(151), 485-494.

Steinemann, S. 1958. Résultats expérimentaux sur la dynamique de la glace et leurs corrélations avec le mouvement et la pétrographie des glaciers. International Association of Scientific Hydrology Publication 47 (Symposium at Chamonix 1958 - Physics of the Movement of the Ice), 184-198.

Taylor, K. C. 1982. Sonic logging at Dye 3, Greenland. (M.Sc. thesis, University of Wisconsin, Madison.)

Thorsteinsson, T. 2000. Anisotropy of ice $\mathrm{I}_{h}$ : development of fabric and effects of anisotropy on deformation. (Ph.D. thesis, University of Washington.)

Thorsteinsson, T. and E. D. Waddington. In press. Folding in strongly anisotropic media. Ann. Glaciol., 35.

Thorsteinsson, Th., J. Kipfstuhl and H. Miller. 1997. Textures and fabrics in the GRIP ice core. f. Geophys. Res., 102(C12), 26,583-26,599.

Thorsteinsson, T., E. D. Waddington, K. C. Taylor, R. B. Alley and D. D. Blankenship. 1999. Strain-rate enhancement at Dye 3, Greenland. 7. Glaciol., 45(150), 338-345.

Van der Veen, G. J. and I. M. Whillans. 1990. Flow laws for glacier ice: comparison of numerical predictions and field measurements. f. Glaciol., 36(124), 324-339.

Van der Veen, C. J. and I. M. Whillans. 1994. Development of fabric in ice. Cold Reg. Sci. Technol., 22(2), 171-195.

Weertman, J. 1963. The Eshelby-Schoeck viscous dislocation damping mechanism applied to the steady-state creep of ice. In Kingery, W. D., ed. Ice and snow: properties, processes, and applications. Cambridge, MA, M.I.T. Press, 28-33.

Wenk, H.-R. and J. M. Christie. 1991. Comments on the interpretation of deformation textures in rocks. F. Struct. Geol., 13(10), 1091-1110.

\section{APPENDIX}

\section{EXPRESSIONS FOR COMPONENTS}

\section{A1. The RSS}

The RSS (Equation (3)) is in general given by

$$
\begin{aligned}
\tau_{(s)}= & n_{1} b_{1}^{(s)} \sigma_{11}+n_{2} b_{2}^{(s)} \sigma_{22}+n_{3} b_{3}^{(s)} \sigma_{33} \\
& +\left(n_{1} b_{2}^{(s)}+n_{2} b_{1}^{(s)}\right) \sigma_{12}+\left(n_{1} b_{3}^{(s)}+n_{3} b_{1}^{(s)}\right) \sigma_{13} \\
& +\left(n_{2} b_{3}^{(s)}+n_{3} b_{2}^{(s)}\right) \sigma_{23},
\end{aligned}
$$

where the three Burgers vectors perpendicular to $\mathbf{n}$ (Equation (11)) are specified as

$$
\begin{aligned}
\mathbf{b}^{(1)}=\frac{1}{3}( & \left.\cos \theta \cos \phi \hat{\mathbf{x}}_{1}+\cos \theta \sin \phi \hat{\mathbf{x}}_{2}-\sin \theta \hat{\mathbf{x}}_{3}\right), \\
\mathbf{b}^{(2)}=-\frac{1}{6}\left((\cos \theta \cos \phi+\sqrt{3} \sin \phi) \hat{\mathbf{x}}_{1}\right. & \left.+(\cos \theta \sin \phi-\sqrt{3} \cos \phi) \hat{\mathbf{x}}_{2}-\sin \theta \hat{\mathbf{x}}_{3}\right), \\
\mathbf{b}^{(3)}=-\frac{1}{6}\left((\cos \theta \cos \phi-\sqrt{3} \sin \phi) \hat{\mathbf{x}}_{1}\right. & \left.+(\cos \theta \sin \phi+\sqrt{3} \cos \phi) \hat{\mathbf{x}}_{2}-\sin \theta \hat{\mathbf{x}}_{3}\right) .
\end{aligned}
$$

The strain rate for a single crystal in uniaxial compression (Equation (14)), using Equations (3), (11) and (A.2), is

$$
\begin{aligned}
\dot{\varepsilon}_{33} & =\frac{1}{2} \sum_{s} 2 \beta \mathcal{A}\left(n_{3} b_{3}^{(s)}\right)\left[n_{3} b_{3}^{(s)} \sigma_{33}\right]^{n} \\
& =\beta \mathcal{A} \sigma_{33}^{3} n_{3}^{4}\left(b_{3}^{(1)^{3}}+b_{3}^{(2)^{3}}+b_{3}^{(3)^{3}}\right),
\end{aligned}
$$


and in simple shear (Equation (21)) it is

$$
\begin{aligned}
\dot{\varepsilon}_{13} & =\frac{1}{2} \beta \mathcal{A} \sum_{s}\left(n_{1} b_{3}^{(s)}+n_{3} b_{1}^{(s)}\right)\left[\left(n_{1} b_{3}^{(s)}+n_{3} b_{1}^{(s)}\right) \sigma_{13}\right]^{n} \\
& =\frac{\beta}{2} \mathcal{A} \sigma_{13}^{3} \sum_{s}\left(n_{1} b_{3}^{(s)}+n_{3} b_{1}^{(s)}\right)^{4} .
\end{aligned}
$$

\section{A2. The velocity gradient in combined uniaxial com- pression and simple shear}

The non-zero velocity gradient components, as a function of stress and cone angle, in combined uniaxial compression and simple shear stress are (with $\mathcal{A}=1$ )

$$
\begin{aligned}
& L_{11}=L_{22}=\sigma^{3}\left\{-64+\cos ^{5}(\alpha)[249-220 \cos (2 \alpha)\right. \\
& +35 \cos (4 \alpha)]\} /[576(\cos (\alpha)-1)] \\
& +\tau^{2} \sigma[-2048+1785 \cos (\alpha)+245 \cos (3 \alpha) \\
& +63 \cos (5 \alpha)+60 \cos (7 \alpha)-105 \cos (9 \alpha)] \\
& /[6144(\cos (\alpha)-1)]
\end{aligned}
$$$$
L_{33}=\left\{6 \sigma \tau^{2}[1024-945 \cos (\alpha)-105 \cos (3 \alpha)\right.
$$$$
+21 \cos (5 \alpha)-30 \cos (7 \alpha)+35 \cos (9 \alpha)]
$$$$
+\sigma^{3}[2048-1890 \cos (\alpha)-420 \cos (3 \alpha)+252 \cos (5 \alpha)
$$$$
+45 \cos (7 \alpha)-35 \cos (9 \alpha)]\} /[9216(\cos (\alpha)-1)] \text {, }
$$

$$
\begin{aligned}
& L_{13}=\left\{\sigma^{2} \tau[-1024+945 \cos (\alpha)+105 \cos (3 \alpha)\right. \\
& -21 \cos (5 \alpha)+30 \cos (7 \alpha)-35 \cos (9 \alpha)] \\
& +\tau^{3}[-3072+2730 \cos (\alpha)-35 \cos (3 \alpha)+357 \cos (5 \alpha) \\
& -15 \cos (7 \alpha)+35 \cos (9 \alpha)]\} /[1536(\cos (\alpha)-1)] .
\end{aligned}
$$

\section{A3. The Johnson model}

The strain-rate components according to Johnson's model are obtained from

$$
\begin{aligned}
& \dot{\varepsilon}_{11}=U\left(\mu \sigma_{11}-\frac{1}{2}(2 \mu-\lambda) \sigma_{22}-\frac{1}{2} \lambda \sigma_{33}\right) \\
& \dot{\varepsilon}_{22}=U\left(-\frac{1}{2}(2 \mu-\lambda) \sigma_{11}+\mu \sigma_{11}-\frac{1}{2} \lambda \sigma_{33}\right), \\
& \dot{\varepsilon}_{33}=U\left(-\frac{1}{2} \lambda\left(\sigma_{11}+\sigma_{22}\right)+\lambda \sigma_{33}\right) \\
& \dot{\varepsilon}_{13}=\frac{1}{2} U \nu \sigma_{13} \\
& \dot{\varepsilon}_{23}=\frac{1}{2} U \nu \sigma_{23} \\
& \dot{\varepsilon}_{12}=\frac{1}{2} U(4 \mu-\lambda) \sigma_{12},
\end{aligned}
$$

where

$$
\begin{aligned}
U & =\frac{1}{2}(2 \mu-\lambda)\left(\sigma_{11}-\sigma_{22}\right)^{2} \\
& +\frac{1}{2} \lambda\left(\left(\sigma_{22}-\sigma_{33}\right)^{2}+\left(\sigma_{33}-\sigma_{11}\right)^{2}\right) \\
& +\nu\left(\sigma_{23}^{2}+\sigma_{13}^{2}\right)+(4 \mu-\lambda) \sigma_{12}^{2} .
\end{aligned}
$$

The Johnson parameters $\lambda, \mu, \nu$ as functions of cone angle (normalized with $\mathcal{A}$ ) are

$$
\begin{aligned}
\lambda^{2} & =(-2048+1890 \cos (\alpha)+420 \cos (3 \alpha)-252 \cos (5 \alpha), \\
& -45 \cos (7 \alpha)+35 \cos (9 \alpha)) /[9216(\cos (\alpha)-1)], \\
\mu^{2} & =(262144-280350 \cos (\alpha)+6300 \cos (3 \alpha) \\
& +13356 \cos (5 \alpha)-225 \cos (7 \alpha)-1225 \cos (9 \alpha)) \\
& /[1179648(1-\cos (\alpha))], \\
\nu^{2} & =2(-3072+2730 \cos (\alpha)-35 \cos (3 \alpha)+357 \cos (5 \alpha) \\
& -15 \cos (7 \alpha)+35 \cos (9 \alpha)) /[3072(\cos (\alpha)-1)] .
\end{aligned}
$$

